\title{
A Residência Médica em Medicina do Trabalho na Faculdade de Medicina da Universidade de São Paulo
}

\author{
The Medical Residency in Occupational Medicine of Medical \\ School of University of São Paulo
}

Rui Manuel Capo', José Wilson Rodrigues de Almeida², Lys Esther Rocha ${ }^{3}$

Capo RM, Almeida JWR, Rocha LE. A residência médica em Medicina do Trabalho na Faculdade de Medicina da Universidade de São Paulo/The medical residency in Occupationl Medicine of Medical School of University of São Paulo. Rev Med (São Paulo). 2011 jul.set.;90(3):108-21.

RESUMO: Objetivo: Descrever as características e a evolução do Programa de Residência Médica em Medicina do TrabaIho na Faculdade de Medicina da Universidade de São Paulo (PMT-FMUSP), desde o seu credenciamento em 2004 até 2010. Método: Estudo descritivo retrospectivo, com base numa revisão bibliográfica de textos institucionais, artigos, e teses e também na análise de documentos arquivados pelo referido Programa e de entrevistas com seus participantes. Resultados: O PMT-FMUSP foi credenciado em 2004 prevendo 5760 horas de treinamento em serviço, divididas em dois anos letivos, com duas vagas. No período de 2004 a 2010, concluíram a residência seis médicos do trabalho. Foram realizadas atividades práticas concentradas nos ambulatórios e serviços de emergência do Hospital das Clínicas da Faculdade, estágios em serviços especializados de empresas privadas de diversos ramos de atividade econômica, sindicatos de trabalhadores e em órgãos públicos relacionados com a saúde dos trabalhadores. As atividades teórico-práticas incluíram aulas no Curso de Especialização de Medicina do Trabalho da Faculdade e reuniões científicas com discussões de casos ou artigos referentes à saúde dos trabalhadores. Conclusão: O PMT-FMUSP é recente e cumpre os requisitos mínimos previstos pela Comissão Nacional de Residência Médica. Ao longo do período em análise, sofreu alterações relacionadas à incorporação de locais de estágios como processo normal de amadurecimento de forma a dotar o médico do trabalho das competências necessárias para o exercício da medicina do trabalho no Brasil.

DESCRITORES: Educação médica; Residência médica; Medicina do trabalho; Saúde ocupacional.
ABSTRACT: Aim: Describe the characteristics and the evolution of the Program of Medical Residency in Occupational Medicine of Medical School of University of São Paulo (PMT-FMUSP), since its accreditation in 2004 until 2010. Method: A retrospective descriptive study based on a bibliographic review of institutional texts, articles and theses and also on the analysis of documents filed by the Program and interviews with the participants. Results: The PMT-FMUSP was accredited in 2004 predicting 5760 hours of service training, divided in two academic years, with two vacancies. Between 2004 and 2010, six occupational doctors graduated. The practical activities were concentrated in the ambulatories and emergency services of the College Hospital, internships in specialized services of private companies which operate in different areas of the economy, labor union and in government agencies related to the workers health. The theorical-practical activities included classes in the Specialization Course of Occupational Medicine of the University and in scientific meetings with discussions about clinical cases or articles about Workers Health. Conclusion: The PMT-FMUSP is recent and fulfills the minimum requirements according to the National Committee of Medical Residency. During the period in analysis, the course suffered changes in its structure, part of a normal process of maturing in a way to give the occupational doctor the necessary abilities for the practice of occupational medicine in Brazil.

KEYWORDS: Internship; Medical residency; Occupational medicine; Occupational health.

1. R2 de Medicina do Trabalho e aluno do Programa Angola - Clínica Multiperfil de capacitação de médicos estrangeiros da Escola de Educação Permanente do Hospital das Clínicas da Faculdade de Medicina da Universidade de São Paulo - HCFMUSP.

2. Docente do Departamento de Medicina Legal, Ética Médica e Medicina Social e do Trabalho da Faculdade de Medicina da Universidade de São Paulo - FMUSP.

3. Supervisora do Curso de Especialização de Medicina do Trabalho da Faculdade de Medicina da Universidade de São Paulo FMUSP.

Endereço para correspondência: Lys Esther Rocha. Departamento de Medicina Legal, Ética Médica, Medicina Social e do Trabalho. Instituto Oscar Freire. Faculdade de Medicina, Universidade São Paulo. Av. Dr. Arnaldo, 455, São Paulo, SP. e-mail: Iysrocha @ usp.br 


\section{INTRODUÇÃO}

Residência Médica constitui
modalidade de ensino de pós-
graduação, destinada à médicos, caracterizada por treinamento em serviço, funcionando sob a responsabilidade de instituições de saúde, universitárias ou não, sob a orientação de profissionais médicos de elevada qualificação ética e profissional'.

Para estabelecer as normas e os requisitos que regulam o credenciamento dos programas em residência médica, foi criada em 1977 a Comissão Nacional de Residência Médica (CNRM) ${ }^{2}$. No Brasil são reconhecidas até o momento 53 especialidades médicas e suas respectivas áreas de atuação. Entre as especialidades reconhecidas pela Comissão Mista de Especialidades está a Medicina do Trabalho (MT).

Para a sua admissão em qualquer programa de Residência Médica, o candidato deverá submeterse ao processo de seleção estabelecido pelo programa aprovado pela CNRM. Os programas de Residência Médica, devido à sua característica de treinamento em serviço, dedicam de $80 \%$ a $90 \%$ da carga horária à estágios em Serviços de Saúde. As atividades teórico-práticas compreendem um mínimo de $10 \%$ e um máximo de $20 \%$ da carga horária total sob a forma de sessões atualizadas, seminários, discussões clínico-patológicas, de acordo com os programas pré-estabelecidos ${ }^{1,7}$.

Segundo Sousa ${ }^{2}$, para oferecer programas de Residência Médica, a instituição interessada deve dispor de infra-estrutura adequada, permitir ao residente a execução de um número suficiente de procedimentos, possuir programa pedagógico bem elaborado e contar com a participação de preceptores preparados e disponíveis para a supervisão das atividades desenvolvidas pelos residentes.

No contexto regional, e de acordo com pesquisa recente ${ }^{3}$ realizada pela Organização PanAmericana de Saúde (OPS) incluindo 14 países da América Latina, a medicina do trabalho é considerada especialidade médica na Argentina, Bolívia, Brasil e Colômbia. Existe ainda como especialidade médica sob a designação de Medicina Ocupacional e do Meio-Ambiente no México e Peru. No Uruguai a especialidade denomina-se Saúde Ocupacional. Não constam na lista de especialidades médicas reconhecidas na República Dominicana, Paraguai, Cuba, Costa Rica, Chile, El Salvador e Honduras.

A Medicina do Trabalho como especialidade médica surgiu com a revolução Industrial, na Europa, na primeira metade do século XIX, época marcada por um acelerado processo de industrialização. $\mathrm{O}$
Dr. Robert Baker primeiro profissional contratado como médico do trabalho, em 1830, na Inglaterra e fundador do primeiro Serviço de Medicina do TrabaIho em uma fábrica têxtil, teorizava que este serviço deveria estar centrado na figura do médico, sendo de sua total responsabilidade todas as ações voltadas a prevenção de danos à saúde, resultantes dos riscos ocupacionais e que o médico do trabalho deveria ser alguém de inteira confiança do empresário ${ }^{4}$.

A recomendação $n^{\circ} 97$ sobre a Proteção da Saúde dos Trabalhadores, organizada pela Organização Internacional do Trabalho (OIT) em 1953, colocava aos países membros a necessidade de fomento da formação de médicos qualificados e a estruturação de Serviços de Medicina do Trabalho nas Empresas ${ }^{4}$.

A Conferência Internacional do Trabalho de 1959 aprovou a recomendação n¹12 sobre "Serviços de Medicina do Trabalho" que se constituiu no primeiro instrumento normativo internacional sobre as atribuições e composição destes serviços e que passou a servir de referência e paradigma para a criação de instrumentos legais, no âmbito nacional dos países membros da $\mathrm{OIT}^{4}$.

No Brasil, a especialidade surge oficialmente em 1972 com a publicação da Portaria $n^{\circ} 3237$ pelo Ministério do Trabalho (revogada em 1978, tendo em vista a revisão do Capítulo $\mathrm{V}$ da Consolidação das Leis do Trabalho (CLT) e a edição da Portaria $\left.n^{\circ} 3214\right)$ que cria os Serviços Especializados em Segurança e Medicina do Trabalho (SESMT) nas empresas através da Norma Regulamentadora $n^{\circ} 4$. Entre os profissionais que compõem estes serviços, está o médico com especialização em medicina do trabalho ${ }^{5,6}$.

A partir dessa legislação, foram criados Cursos de Especialização em Medicina do Trabalho por instituições de ensino, sob a supervisão da Fundação Jorge Duprat de Figueiredo de Segurança e Medicina do Trabalho (FUNDACENTRO), órgão educativo e de pesquisa do Ministério do Trabalho.

Tais cursos tiveram como objetivo atender a demanda de profissionais para cumprir a exigência legal que tornou obrigatória a organização dos SESMT nas empresas, fazendo com que num curto período de tempo fosse preparado um considerável número de médicos capacitados, para implementar a nova legislação ${ }^{\text {. }}$.

Na Faculdade de Medicina da Universidade de São Paulo (FMUSP), a formação do Médico do Trabalho, iniciou-se na década de 1980, sob a forma de Curso de Especialização, com a supervisão do Ministério do Trabalho, vinculado ao Departamento de Medicina Legal, Ética Médica e Medicina Social e do Trabalho. 
A formação do especialista em medicina do trabalho na modalidade de Residência Médica foi iniciada nos anos 80, no bojo do movimento da Medicina Social. Estas Residências foram organizadas no âmbito dos Departamentos de Medicina Preventiva e Social, ou de Saúde Comunitária, como uma área de concentração em Saúde Ocupacional, nas residências em Medicina Social ou Medicina Comunitária 5 . Neste período, o Departamento de Medicina Preventiva da FMUSP, oferecia a possibilidade do terceiro ano da Residência Médica em Medicina Preventiva ser realizado em Saúde Ocupacional.

Em 2002, através da resolução 1634, o Conselho Federal de Medicina (CFM), a Associação Médica Brasileira (AMB) e a CNRM formaram a Comissão Mista de Especialidades (CME), que reconheceu a Medicina do Trabalho como especialidade médica ${ }^{7}$.

Segundo Silveira e Dias ${ }^{7}$ a partir deste reconhecimento as instituições de ensino estruturaram os Programas de Residência Médica em Medicina do Trabalho. Os Programas foram organizados de modo que o médico residente cumpra um conjunto de atividades que lhe confira competências específicas para o exercício profissional.

Dias et al. ${ }^{6}$ enumeram as competências básicas para o médico especialista em Medicina do Trabalho: (1) Estudo do trabalho; (2) Atenção integral (individual e coletiva) à saúde do trabalhador; (3) Elaboração e execução de políticas de gestão da saúde no trabalho; (4) Estabelecer protocolos, normas e procedimentos e (5) Produção e divulgação de conhecimentos técnicos - educação permanente.

Para a aquisição de tais competências os médicos residentes devem, durante a sua formação, estudar os processos de trabalho para conhecer os aspectos técnicos e organizacionais e avaliar e controlar os fatores de risco; desenvolver ações de atenção e assistência à saúde dos trabalhadores em forma de estágios na rede pública de serviços de saúde, nos SESMT de empresas e serviços de saúde de sindicatos de trabalhadores; avaliar incapacidade para o trabalho e promover ações de reabilitação; desenvolver atividades educativas, formação, capacitação e vigilância da saúde do trabalhador; participar do planejamento e gestão dos serviços de saúde do trabalhador bem como na formulação de políticas em saúde do trabalhador ${ }^{6}$.

Além destes itens, os médicos residentes participam de atividades acadêmicas, seminários e cursos, visando o aprofundamento teórico e a aquisição de habilidades em temas específicos ${ }^{7}$.

Atualmente há duas formas de obter-se o reconhecimento como Especialista em Medicina do Trabalho pelo CFM: ter concluído a Residência
Médica em instituição credenciada pela CNRM ou ser portador do Título de Especialista emitido pela AMB/ANAMT. Para a participação na prova de título de especialista em Medicina do Trabalho é exigida a formação em treinamento de dois anos em serviço na especialidade, com carga horária total de 3.840 horas ou o exercício da especialidade de medicina do trabalho por quatro anos ${ }^{8}$.

O título de especialista em Medicina do Trabalho é considerado pela ANAMT como uma das melhores formas de mostrar a toda sociedade e à comunidade médica, em particular, a qualificação profissional necessária para atuar com competência na área médica relacionada à saúde e segurança do trabalho. A prova de titulo da ANAMT obedece a um regulamento que valoriza a participação do médico em eventos científicos, experiência profissional, atividades e programas de treinamento específicos e atualização continuada, tendo por base todas as competências requeridas para o exercício da Medicina do Trabalho. Esta prova é realizada com uma periodicidade mínima anual. Geralmente a prova ocorre durante um evento científico da ANAMT, como Congressos Nacionais ou Seminários Regionais ${ }^{8}$.

No Brasil, devido ao recente reconhecimento da especialidade, existem poucos estudos caracterizando o funcionamento e avaliação dos seus programas de residência ${ }^{9,10}$.

Na FMUSP, o Programa de Residência Médica em Medicina do Trabalho foi implantado em 2004. Desta forma, este estudo teve como objetivo analisar e descrever as características do Programa de Residência Médica em Medicina do Trabalho na FMUSP desde a sua criação até 2010 , estabelecendo as diferenças e semelhanças com os requisitos mínimos propostos pela CNRM, caracterizando as atividades teóricas e práticas dos médicos residentes; analisando os estágios em áreas clínicas, empresas públicas e privadas.

\section{MÉTODOS}

Foi feito um estudo descritivo retrospectivo analisando o programa de residência médica em Medicina do Trabalho da FMUSP no período de 2004 à 2010, em três fases.

\section{Fase I - Revisão bibliográfica}

Revisão sobre o tema com consulta de artigos, teses, dissertações nas revistas disponíveis nas bases de dados Pubmed, SciELO, Lilacs. Foram lidos os resumos do material bibliográfico descrito acima e na íntegra os que tinham relação com o tema e os objetivos propostos neste estudo. 
Nestas bases de dados foram encontrados cinco estudos sobre residência médica em Medicina do Trabalho e outros cinco que caracterizavam as residências médicas em outras especialidades. Estes serviram de base para a construção da metodologia, enquanto que aqueles foram usados para comparação dos resultados.

Foram consultadas outras fontes com dados sobre Legislação do Ministério da Educação e Cultura; documentos da CNRM, site da ANAMT e temas livres sobre Residência dos Congressos da ANAMT/ Conferência Latino Americana de Saúde Ocupacional e Organização Panamericana de Saúde (OPS).

Fase II - Diagnóstico da residência médica em medicina do trabalh da FMUSP

Foi analisado o Programa de Residência Médica em Medicina do Trabalho da FMUSP em relação ao credenciamento, grade curricular, carga horária teórica e prática, locais e duração dos estágios desde a sua criação em 2004 até 2010 por meio de análise dos seguintes documentos:

> Pedido de credenciamento em 2004 e recredenciamento em 2009 da residência médica em MT; programa;

$>$ Documentos da CNRM fornecidos ao

> Programas anuais de estágios da Residência Médica em MT através dos formulários de avaliação individual dos residentes, disponíveis na Comissão de Residência Médica (COREME) da FMUSP no período;

> Relatórios de avaliação dos residentes sobre os estágios arquivados pelo programa;

> Entrevistas informais com os residentes, preceptores e supervisores quanto aos locais de estágios e programa de atividades teórico-práticas no período.

Fase III - Análise dos dados e das fontes de informações

Os documentos arquivados no Departamento de Medicina Legal, Ética Médica e Medicina Social e do Trabalho da FMUSP, no site e arquivos da Comissão de Residência Médica (COREME), serviram de base para a obtenção das informações referentes ao credenciamento do Programa de Residência Médica de Medicina do Trabalho da FMUSP e dos diversos programas de estágios dos médicos residentes no período em estudo. Estes documentos permitiram comparar as características do programa em relação aos requisitos mínimos da CNRM.
Recorremos igualmente aos arquivos da Comissão de Residência Médica, para analisarmos as opiniões dos preceptores através dos formulários de avaliação dos residentes, preenchidos no final de cada estágio. Os formulários são enviados à COREME, com cópias arquivadas no Departamento e foram usados para identificar as características dos residentes que concluíram o programa quanto a idade, sexo e procedência e também os estágios realizados em áreas clínicas e empresas.

Além do formulário de avaliação preenchido pelos preceptores, alguns médicos residentes também avaliaram os seus estágios através de relatórios, nos quais forneceram suas opiniões sobre a pertinência da presença dos estágios na grade do residente do ano seguinte bem como sua carga horária.

As entrevistas informais com os residentes, preceptores e supervisores do Programa de Residência Médica de Medicina do Trabalho da FMUSP, serviram para obter informações não arquivadas formalmente no Departamento, nem nos arquivos da COREME, mas que representavam dados importantes no histórico do processo de credenciamento da residência, na seleção dos médicos candidatos a residentes, na construção e evolução cronológica das atividades teóricas e práticas.

As informações obtidas serão apresentadas em tabelas usando o programa Microsoft Excel 12.0 (Office 2007).

\section{RESULTADOS}

\section{Histórico do processo de credenciamento}

\section{Comparação do programa mínimo da CNRM com o programa da FMUSP}

O processo de credenciamento do Programa de Residência Médica em Medicina do Trabalho na FMUSP iniciou-se em 28 de junho de 2002 com o convite da COREME ao Departamento de Medicina Legal, Ética Médica e Medicina Social e do Trabalho para a elaboração do Programa de Residência Médica na especialidade.

Em 14 de fevereiro de 2003, foi encaminhado o Programa Oficial de Credenciamento da Residência Médica em Medicina do Trabalho à CNRM, com proposta inicial para um total de quatro residentes, sendo dois para o primeiro ano (R1) e dois para o segundo ano (R2). A solicitação de credenciamento incluía uma grade curricular para o primeiro ano da residência (R1) dividida em: estudos dos processos de trabalho; atenção à saúde do trabalhador; atividades 
educativas em saúde do trabalhador; vigilância à Saúde do Trabalhador. A grade curricular proposta para o R1 incluía também atividades científicas com discussão de casos clínico-periciais, artigos científicos em reuniões semanais bem como aulas teóricas e práticas no curso de especialização em Medicina do Trabalho com 2880h.

A Tabela 1 compara os requisitos mínimos exigidos pela CNRM com a proposta de programa apresentada pelo Departamento de Medicina Legal, Ética Médica e Medicina Social e do Trabalho para o R1 de Medicina do Trabalho da FMUSP, mostrando que o programa cumpriu os requisitos mínimos com ênfase em atividades clínicas referentes à atenção à saúde do trabalhador, desenvolvidas no Hospital das Clínicas da FMUSP.

Tabela 1. Requisitos mínimos da CNRM X Proposta de programa para credenciamento (R1 de Medicina do Trabalho da FMUSP, 2004)

\begin{tabular}{|c|c|c|}
\hline \multicolumn{2}{|c|}{ Requisitos da Comissão Nacional de Residência Médica } & \multirow{2}{*}{$\frac{\text { Proposta da FMUSP (2004) }}{\text { Serviços }}$} \\
\hline Atividade/ hr ano & Serviços & \\
\hline Estudo dos processos de trabalho & $\begin{array}{l}\text { Serviço de Saúde do Trabalhador, } \\
\text { Sitema Único de Saúde (SUS) } \\
\text { SESMT públicos e privados }\end{array}$ & $\begin{array}{l}\text { Ambulatórios de grandes empresas } \\
\text { e serviços de assessoria médicas }\end{array}$ \\
\hline $\begin{array}{l}\text { Avaliação e controle de fatores de risco } \\
(20 \%)\end{array}$ & $\begin{array}{l}\text { Prestadores de serviços de Saúde } \\
\text { e Segurança e Sindicatos }\end{array}$ & $\begin{array}{l}\text { em Saúde ocupacional } \\
(432 h)(15 \%)\end{array}$ \\
\hline Atenção a saúde do trabalhador & Unidade Básica de Saúde (SUS) & Ambulatório Geral Didático (AGD) \\
\hline \multirow{6}{*}{$\begin{array}{l}\text { Promoção, prevenção e proteção a saúde, } \\
\text { diagnóstico, tratamento e reabilitação (45\%) }\end{array}$} & Unidades de urgência e emergência & Ambulatório das especialidades clínicas \\
\hline & $\begin{array}{l}\text { Cuidados secundários e terciários } \\
\text { em saúde do Trabalhador }\end{array}$ & Centro de Saúde Escola Samuel Pessoa \\
\hline & & $\begin{array}{l}\text { Centro de Promoção à Saúde (CPS) } \\
\text { Pronto Socorro e Unidade de Terapia } \\
\text { Intensiva de Clínica Médica do HCFMUSP } \\
\text { Pronto Socorro de Ortopedia do HCFMUSP }\end{array}$ \\
\hline & & $\begin{array}{l}\text { Serviço de Saúde Ocupacional (SSO) do } \\
\text { HCFMUSP }\end{array}$ \\
\hline & & $\begin{array}{l}\text { Divisão de Medicina de Reabilitação da } \\
\text { FMUSP }\end{array}$ \\
\hline & & $(1440 h)(50 \%)$ \\
\hline \multirow{3}{*}{$\begin{array}{l}\text { Atividades educativas em saúde do } \\
\text { trabalhador (10\%) }\end{array}$} & $\begin{array}{l}\text { Rede de serviços de saúde do } \\
\text { trabalhador }\end{array}$ & $\begin{array}{l}\text { Fundação Jorge Duprat de Figueiredo de } \\
\text { Medicina do Trabalho (FUNDACENTRO) }\end{array}$ \\
\hline & $\begin{array}{l}\text { do Sistema Único de Saúde (SUS), } \\
\text { FUNDACENTRO, Entidades }\end{array}$ & $(144 h)(5 \%)$ \\
\hline & $\begin{array}{l}\text { Patronais/ Organizaçoes de } \\
\text { trabalhadores }\end{array}$ & \\
\hline Vigilância à saúde do trabalhador (10\%) & $\begin{array}{l}\text { Centro de Referência de Saúde do } \\
\text { Trabalhador (CRST) } \\
\text { Superintendência Regional do } \\
\text { Trabalho (SRT) }\end{array}$ & $\begin{array}{l}\text { Centro de Referência de Saúde do } \\
\text { Trabalhador (CRST) } \\
\text { Superintendência Regional do Trabalho } \\
\text { (SRT) }(288 \text { h) }(10 \%)\end{array}$ \\
\hline \multirow[t]{5}{*}{ Cursos obrigatórios } & & Reunião geral da clínica médica \\
\hline & & Reunião científica da clínica médica \\
\hline & & Reunião anátomo-patológica \\
\hline & & Discussão de casos clínicos/periciais \\
\hline & & Discussão de artigos científicos \\
\hline \multirow[t]{3}{*}{$\begin{array}{l}\text { Controle de infecção hospitalar, epidemio- } \\
\text { logia, bioestatística metodologia científica, } \\
\text { ergonomia, ética médica, bioética ( } 20 \%)\end{array}$} & & $\begin{array}{l}\text { Cursos: controle de infecção hospitalar, } \\
\text { epidemiologia, bioestatística, metodologia } \\
\text { científica, }\end{array}$ \\
\hline & & $\begin{array}{l}\text { fundamentos de ergonomia, ética médica, } \\
\text { bioética }\end{array}$ \\
\hline & & $(576 h)(20 \%)$ \\
\hline
\end{tabular}

Fonte: Arquivos do Departamento de Medicina Legal, Ética Médica, Medicina Social e do Trabalho da FMUSP, 2004-2010. 
Para o segundo ano (R2), propôs-se uma grade curricular que incluía atividades de atenção e assistência à saúde do trabalhador; avaliação de incapacidade para o trabalho e reabilitação; planejamento, gestão de serviços de saúde do trabalhador e elaboração de políticas; atividades educativas, formação e capacitação em saúde do trabalhador. Na Tabela 2 compara-se essa grade curricular com os requisitos da CNRM.

Tabela 2. Requisitos mínimos da CNRM X Proposta de programa para credenciamento (R2 de Medicina do Trabalho da FMUSP, 2004)

\begin{tabular}{|c|c|c|}
\hline \multicolumn{2}{|c|}{ Requisitos da Comissão Nacional de Residência Médica } & \multirow{2}{*}{$\begin{array}{r}\text { Proposta da FMUSP (2004) } \\
\text { Seviços }\end{array}$} \\
\hline Atividade/ $\mathrm{h} /$ ano & Serviços & \\
\hline $\begin{array}{l}\text { Atenção à saúde do trabalhador/ } \\
\text { assistência } \\
\text { Promoção e proteção da saúde } \\
\text { Prevenção, Diagnóstico, Tratamento e } \\
\text { Reabilitação (20\%) }\end{array}$ & $\begin{array}{l}\text { Cuidados secundário e terciário em } \\
\text { saúde do trabalhador }\end{array}$ & $\begin{array}{l}\text { Ambulatório de Especialidades: Psiquiatria; } \\
\text { Ortopedia e Traumatologia; Dermatologia; } \\
\text { Otorrinolaringologia; Oftalmologia } \\
(576 \mathrm{~h})(20 \%)\end{array}$ \\
\hline $\begin{array}{l}\text { Avaliação da incapacidade para o trabalho } \\
\text { e reabilitação (10\%) }\end{array}$ & $\begin{array}{l}\text { Perícia e reabilitação do INSS; } \\
\text { outros órgãos públicos }\end{array}$ & $\begin{array}{l}\text { Divisão de Medicina de Reabilitação } \\
\text { Centro de Reabilitação do HCFMUSP } \\
\text { Instituto de Medicina Criminalística do Estado } \\
\text { de São Paulo (IMESC) } \\
\text { Serviço de Perícia Médica do Instituto Nacional } \\
\text { de Seguro Social (INSS) (288h) (10\%) }\end{array}$ \\
\hline $\begin{array}{l}\text { Planejamento, gestão de serviços de } \\
\text { saúde do trabalho e elaboração de } \\
\text { políticas }(20 \%)\end{array}$ & $\begin{array}{l}\text { Coordenação de Saúde do } \\
\text { Trabalhador do SUS; SESMT de } \\
\text { empresas públicas e privadas; } \\
\text { Prestadores de serviços de saúde e } \\
\text { segurança e Sindicatos }\end{array}$ & $\begin{array}{l}\text { Ambulatório do Serviço de Saúde Ocupacional } \\
\text { (SSO) do HCFMUSP } \\
\text { Ambulatório de grandes empresas e serviços } \\
\text { de assessoria médica em saúde ocupacional } \\
(720 h)(25 \%)\end{array}$ \\
\hline $\begin{array}{l}\text { Atividades educativas, formação e } \\
\text { capacitação em saúde do trabalhador } \\
(10 \%)\end{array}$ & $\begin{array}{l}\text { Rede de serviços de saúde } \\
\text { do trabalhador do SUS e } \\
\text { FUNDACENTRO } \\
\text { Entidades patronais/ sindicatos }\end{array}$ & $\begin{array}{l}\text { SRT; FUNDACENTRO; CRST } \\
\text { Serviço de saúde dos sindicatos } \\
(720 \mathrm{~h})(25 \%)\end{array}$ \\
\hline $\begin{array}{l}\text { Cursos obrigatórios } \\
\text { Cursos e estágios optativos }\end{array}$ & $\begin{array}{l}\text { Higiene do Trabalho } \\
\text { Toxicologia }\end{array}$ & $\begin{array}{l}\text { Curso de especialização de Medicina do } \\
\text { Trabalho }\end{array}$ \\
\hline $\begin{array}{l}\text { Psicodinâmica do trabalho; } \\
\text { Poluição ambiental e saneanento do meio; } \\
\text { Gestão integrada de saúde; } \\
\text { Segurança e meio ambiente; } \\
\text { Processos de certificação; } \\
\text { Promoção da Saúde no Trabalho; } \\
\text { Laboratório de Toxicologia Ocupacional; } \\
\text { Dermatologia Oupacional; } \\
\text { Pneumopatia Ocupacional; } \\
\text { Hematologia; } \\
\text { Otorrinolaringologia ocupacional; } \\
\text { Neurologia e Neurotoxicologia } \\
\text { Ocupacional (20\%) }\end{array}$ & Segurança do Trabalho & $\begin{array}{l}\text { Laboratório de Toxicologia e Genética } \\
\text { Molecular } \\
\text { Discussão de casos clínicos/ perícias } \\
\text { Discussão de artigos científicos }\end{array}$ \\
\hline
\end{tabular}

Legenda: CRST: Centro de Referência de Saúde do Trabalhador; INSS: Instituto Nacional de Seguro Social; SESMT: Serviço Especializado de Engenharia de Segurança e Medicina do Trabalho; SRT: Superintendência Regional do Trabalho; SUS: Sistema Único de Saúde.

Fonte: Arquivos do Departamento de Medicina Legal, Ética Médica, Medicina Social e do Trabalho da FMUSP, 2004-2010. 
No total, a proposta de programa era de $5.760 \mathrm{~h}$ de treinamento em serviço com atividades no primeiro e segundo ano.

Para efeito de transição do primeiro para o segundo ano e conclusão da residência médica em Medicina do Trabalho, os médicos residentes seriam avaliados através de: avaliações dos estágios pelos preceptores e assistentes; relatório de aproveitamento do estágio elaborado pelo residente; provas das disciplinas do curso de especialização de MT da FMUSP; relatórios das visitas às empresas no curso de especialização de MT; trabalho de conclusão de curso (TCC) apresentado no final da residência.

Após a apresentação dessa proposta de programa para a Residência Médica em Medicina do Trabalho à Comissão Nacional de Residência Médica, a instituição foi vistoriada em 20 de agosto de 2003 e foi considerada apta a atender as recomendações deste órgão do Ministério da Educação e Cultura (MEC), quer na distribuição da carga horária bem como no conteúdo programático a ser desenvolvido pelos residentes (Of. 324/2003/ COREME). O credenciamento foi confirmado em 2 de outubro de 2003 através de parecer conclusivo do plenário da CNRM n ${ }^{\circ}$ 159/03.

Tendo em vista o prazo de validade do primeiro credenciamento em 2004 vencido, em junho de 2009 foi enviada à CNRM uma nova proposta de (re) credenciamento do programa, cujo conteúdo em termos de áreas de concentração dos estágios para $\mathrm{R} 1$ e $\mathrm{R} 2$ e respectivas cargas horárias eram basicamente as mesmas de 2004.

\section{Histórico do processo seletivo}

Durante os dois anos de residência médica, os médicos residentes são remunerados através de uma bolsa de estudo paga pela FUNDAP (Fundação do Desenvolvimento Administrativo do Estado de São Paulo), cujo valor é estabelecido pela CNRM. Para o programa de residência médica em MT da FMUSP foram credenciadas duas vagas pela CNRM, mas apenas cedida uma bolsa por ano pela COREME.

Como previsto no programa, os médicos candidatos inscritos para o primeiro ano da residência, foram selecionados mediante processo que incluiu provas teóricas e práticas, inseridas na programação geral anual de admissão de novos residentes para o HCFMUSP e entrevistas com os supervisores do programa de Medicina do Trabalho.

No total, foram seis egressos do programa, sendo três do sexo masculino e três do sexo feminino, com idade média de 29 anos e procedentes de várias regiões do país, mostrando a heterogeneidade dos médicos que concluíram a residência de Medicina do Trabalho da FMUSP de 2004 à 2010, quanto a procedência e locais de graduação.

Em 2004 foi matriculado um médico residente de 25 anos, natural de Recife, com graduação concluída em 2003 na Universidade Federal de Pernambuco (UFPE). Para o ano de 2005, o programa matriculou para o primeiro ano da residência dois médicos, sendo uma de 29 anos, natural de Joinville, com formatura em 2002 na Universidade Federal de Santa Catarina (UFSC) e outro de 35 anos, natural de São Paulo, com graduação na USP em 2001. Nesse ano, uma das bolsas foi paga por uma empresa. Esta experiência mostrou-se ineficaz já que, no decorrer do ano letivo, houve aumento do valor da bolsa, resultado do movimento de reivindicação por parte dos médicos residentes no país e a empresa patrocinadora não se disponibilizou para cobrir o valor adicional, tendo-se decidido não mais repetir a experiência nos anos seguintes.

No ano de 2006, nenhum candidato foi matriculado para freqüentar o primeiro ano da residência, por desistência dos dois primeiros classificados no processo seletivo e atraso na matrícula do terceiro candidato. A bolsa da MT deste ano foi perdida para outro programa de residência da FMUSP.

Em 2007 uma residente de 27 anos e proveniente de São Paulo foi matriculada. Ela concluiu a graduação em 2005 na Faculdade de Medicina do ABC (FMABC).

Para o ano letivo de 2008, matriculou-se no primeiro ano uma residente de 28 anos, natural de São Paulo e graduada na Universidade Estadual Paulista (UNESP) em 2005 e no último ano do período em análise (2009), igualmente um residente foi matriculado para freqüentar o primeiro ano da residência médica em $M T, 29$ anos e natural de Taubaté, São Paulo, com graduação em 2006 na Universidade Federal de Juiz de Fora (UFJF).

\section{Descrição do programa de atividades}

Atividades práticas: estágios no Hospital das Clínicas da FMUSP

A Tabela 3 apresenta os estágios na área clínica dos residentes do primeiro (R1) e segundo anos (R2) de Medicina do Trabalho, todos eles desenvolvidos no HC da FMUSP. Estes estágios tiveram como objetivo a formação em clinica médica necessária para o exercício da especialidade. 
Capo et al. A Residência Médica em Medicina do Trabalho na FMUSP.

Tabela 3. Distribuição das áreas clínicas de estágio/ residentes (R1 e R2) em Medicina do Trabalho da FMUSP, 2004-2010

\begin{tabular}{|c|c|c|c|c|c|c|}
\hline Áreas de estágio & 2004-2005 & 2005-2006 & 2005-2006 & 2007-2008 & 2008-2009 & 2009-2010 \\
\hline Pronto Socorro da Clínica Médica (PSM) & $x$ & $x$ & $x$ & $x$ & $x$ & $x$ \\
\hline Unidade de Terapia Intensiva (UTI) & $x$ & $x$ & $x$ & $\mathrm{x}$ & $x$ & $\mathrm{x}$ \\
\hline Ambulatório Geral e Didático (AGD) & $x$ & $\mathrm{X}$ & $\mathrm{x}$ & $x$ & $x$ & $\mathrm{X}$ \\
\hline Divisão de Medicina de Reabilitação (DMR) & $x$ & & & & $x$ & $x$ \\
\hline Instituto de Ortopedia e Traumatologia (IOT) & $\mathrm{X}$ & $x$ & $x$ & $x$ & $\mathrm{X}$ & $\mathrm{x}$ \\
\hline Pronto Socorro de Cirurgia (PSC) & $x$ & $x$ & $x$ & $x$ & $x$ & $x$ \\
\hline Moléstias Infecciosas e Parasitárias (MIP) & & $x$ & & & & \\
\hline Dermatologia & $x$ & & & & & $\mathrm{x}$ \\
\hline Pneumologia & $x$ & $x$ & $x$ & $x$ & $x$ & $x$ \\
\hline Otorrinolaringologia (ORL) & $\mathrm{X}$ & & & $\mathrm{x}$ & & $\mathrm{x}$ \\
\hline Serviço de Saúde Ocupacional (SSO) & $x$ & $x$ & $\mathrm{x}$ & $x$ & $x$ & $x$ \\
\hline Neurologia & & & & $x$ & $x$ & $x$ \\
\hline Oftalmologia & & & & $x$ & $x$ & $x$ \\
\hline Instituto de Psiquiatria (IPq) & & $x$ & & & & \\
\hline
\end{tabular}

Fonte: Formulário de avaliação individual de residente, COREME 2004-2010.

No primeiro ano, buscou-se a inserção em ambulatórios de diferentes especialidades e serviços de urgência e no segundo ano, foram selecionados ambulatórios específicos de Saúde Ocupacional como Pneumologia, Otorrinolaringologia, Dermatologia, dentre outros.

De forma geral todos os residentes, desde o início da residência em 2004, passaram pelos ambulatórios de especialidades e serviços de atendimento de urgência do Hospital das Clínicas da FMUSP. O estágio em Psiquiatria foi feito no Instituto de Psiquiatria (IPq) em 2005/2006 e desde então passou a estar integrado no Ambulatório Geral e Didático (AGD) da Clínica Médica do HCFMUSP. O Serviço de Moléstias Infecciosas e Parasitárias (MIP) foi área de estágio apenas para o R1 no ano letivo 2005/2006.

\section{Estágios em serviços especializados de empresas} públicas e privadas e sindicatos de trabalhadores

O estágio nos serviços de saúde das grandes empresas públicas e privadas e sindicatos de trabalhadores tem como objetivo estudar e avaliar os processos de trabalho, as condições ambientais dos locais de trabalho, as formas de organização do trabalho e sua relação com os fatores de adoecimento dos trabalhadores, atividades educativas, de planejamento, gestão e organização dos SESMT e das empresas no geral, bem como avaliar a percepção dos trabalhadores e suas organizações sindicais, a respeito da sua saúde.

Ao longo do período em análise, foram selecionadas várias empresas, tendo como critério a diversidade e complexidade dos seus respectivos ramos de atividade, para dar aos futuros médicos do trabalho visões diferentes de processos de trabalho, riscos ocupacionais, ações educativas, de promoção e prevenção.

Durante os três primeiros anos de existência da residência de MT da FMUSP, os estágios em empresas concentraram-se nos ramos hospitalar, automobilístico, petroquímico, metalúrgico e serviços de saúde de sindicatos de trabalhadores. Os ramos de transporte aéreo, indústria alimentar e teleatendimento foram introduzidos apenas em 2007. Os serviços de saúde do sindicato de trabalhadores e o ramo automobilístico estiveram presentes na grade de estágio até 2006 (Tabela 4). 
Tabela 4. Distribuição das áreas de estágio em empresas por ramo de atividade e Serviços de Saúde de Sindicatos de trabalhadores para os residentes (R1 e R2) em MT da FMUSP, 2004-2010

\begin{tabular}{|c|c|c|c|c|c|c|}
\hline Áreas de estágio & 2004-2005 & 2005-2006 & $2005-2006$ & 2007-2008 & 2008-2009 & $2009-2010$ \\
\hline Metalúrgicas & $x$ & $x$ & & $x$ & $x$ & \\
\hline Automobilística & $x$ & $x$ & $x$ & & & \\
\hline Serviço de saúde hospitalar & $x$ & $x$ & $x$ & $x$ & $x$ & $x$ \\
\hline Petroquímica & $x$ & $x$ & $x$ & $x$ & & \\
\hline Empresa tranporte aéreo & & & & $x$ & $x$ & $x$ \\
\hline Indústria alimentícia & & & & $x$ & $x$ & $x$ \\
\hline Teleatendimento & & & & $x$ & $x$ & $x$ \\
\hline Laboratório de Toxicologia & & $x$ & $x$ & & & \\
\hline Serviço de Saúde do Sindicato (SSS) & $x$ & $x$ & $x$ & & & \\
\hline
\end{tabular}

Fonte: Formulário de avaliação individual de residente, COREME 2004-2010.

Estas diferenças dos locais de estágio estão relacionadas à disponibilidade das empresas na aceitação dos residentes e na garantia de supervisão adequada nos locais de estágio.

Entre os residentes que concluíram suas atividades, tivemos uma residente que efetuou estágios em Serviços de Saúde dos Trabalhadores da Itália e Bélgica.

\section{Estágios em serviços públicos de saúde dos trabalhadores}

Na Tabela 5 estão representados os estágios nos serviços públicos selecionados para os residentes de Medicina do Trabalho da FMUSP, tendo como objetivo, conhecer ações de inspeção do trabalho e ações interdisciplinares em saúde e trabalho, atividades educativas e de pesquisa e as políticas de gestão em saúde do trabalhador.

Durante o período em análise, todos os residentes tiveram oportunidade de passar pela Superintendência Regional do Trabalho (SRT) e Fundação Jorge Duprat de Figueiredo de Segurança e Medicina do Trabalho (FUNDACENTRO) ambos, órgãos do Ministério do Trabalho e Emprego (MTE), bem como nos Serviços de Saúde do Trabalhador do Ministério da Saúde (MS), nomeadamente a
Secretaria de Estado da Saúde (SES) e os Centros de Referência a Saúde do Trabalhador (CRST/ CEREST), para atenção secundária e planejamento e gestão em saúde do trabalhador.

Da mesma forma o Centro de Saúde Escola (CSE) Samuel Pessoa, instituição de atenção primária do Sistema Único de Saúde (SUS) ligada à FMUSP, constituiu área de estágio para todos os residentes de MT da FMUSP desde 2004, tem como objetivo dar aos residentes a oportunidade de aprofundarem conhecimentos sobre a forma de organização da assistência do SUS, articulação entre os três níveis de atenção à saúde e abordarem a saúde do trabalhador no nível básico e suas interfaces com o Programa de Saúde da Família (PSF), Programa de Saúde Mental, Saúde do Idoso, Programa de Saúde da Mulher, Saúde Mental e outros ${ }^{11}$.

O Instituto Nacional de Segurança Social (INSS), instituição previdenciária de concessão de benefícios aos trabalhadores afastados por incapacidade para o trabalho, constituiu uma área importante de estágio para os médicos residentes de medicina do trabalho. Durante o período em análise não esteve presente na grade curricular dos anos letivos de 2004/ 2005 e 2005/2006.

Quanto ao Instituto de Medicina Social e de Criminologia do Estado de São Paulo (IMESC) fez parte da grade de estágio dos residentes apenas em 2007/2008 (Tabela 5). 
Tabela 5. Distribuição das áreas de estágio em órgãos públicos por residentes (R1 e R2) em MT da FMUSP, 20042010

\begin{tabular}{lcccccc}
\hline Áreas de estágio & 2004-2005 & 2005-2006 & 2005-2006 & 2007-2008 & 2008-2009 & 2009-2010 \\
DRT & $X$ & $X$ & $X$ & $X$ & $X$ \\
Fundacentro & $X$ & $X$ & $X$ & $X$ & $X$ & $X$ \\
INSS & & $X$ & $X$ & $X$ & $X$ \\
IMESC & $X$ & $X$ & $X$ & $X$ \\
SES/CEREST/CRST & $X$ & $X$ & $X$ & $X$ & $X$ \\
CSE & $X$ & $X$ & $X$ & $X$ \\
\hline
\end{tabular}

Legenda: DRT: Delegacia Regional do Trabalho; FUNDACENTRO: Fundação Jorge Duprat de Figueiredo de Segurança e Medicina do Trabalho; INSS: Instituto Nacional se Seguro Social; IMESC: Instituto de Medicina Social e Criminologia do Estado de São Paulo; SES: Secretaria Estadual e Saúde; CEREST: Centro Estadual de Referência de Saúde do Trabalhador; CRST: Centro de Referência de Saúde do Trabalhador; CSE: Centro de Saúde Escola.

Fonte: Formulário de avaliação individual de residente, COREME 2004-2010.

Atividades teórico-práticas

As atividades teórico-práticas incluíram discussões de casos clínicos e artigos científicos, versando temas sobre os principais agravos à saúde dos trabalhadores, cursos e estágios optativos, bem como a elaboração de uma monografia como trabalho de conclusão de curso.

Durante o período em análise, foram realizadas e registradas atas de 46 reuniões de residentes e supervisores do programa de MT da FMUSP e convidados. Destas, 36 foram para discussão de casos clínicos, artigos científicos e temas diversos em saúde do trabalhador, apresentados pelos residentes ou convidados especiais.

Estas atividades iniciaram ações conjuntas com a Residência de Medicina Legal da FMUSP em 2004 sob a forma de reuniões mensais. A partir de 2007, as reuniões passaram a ser quinzenais e, desde 2008, semanais.

A preceptoria da residência de Medicina do Trabalho é feita em conjunto com a residência de Medicina Legal de forma alternada desde 2007, com uma bolsa disponibilizada para o Departamento. No período em análise os residentes da Medicina do Trabalho assumiram a preceptoria somente em 2009.

Além disso, os residentes da Medicina do Trabalho da FMUSP são incentivados a participar de cursos, seminários, jornadas, congressos e em outros fóruns em que a saúde dos trabalhadores é abordada, quer como participantes ou como expositores.
Como exemplo, o último médico residente (2010) além da residência de Medicina do Trabalho, freqüentou também o Curso de Especialização de Medicina Legal e Perícias Médicas e de Medicina do Tráfego da Faculdade de Medicina da Universidade de São Paulo; participou do Curso de Inclusão de Pessoas com Deficiência no Mercado de Trabalho e Curso de Ergonomia e Segurança na Empresa; Workshop da OIT sobre Experiências e Práticas na Prevenção de Grandes Acidentes; Nexo Técnico Epidemiológico e Fator Acidentário de Prevenção como Instrumentos de Proteção à Saúde dos Trabalhadores, todos pela Fundação Jorge Duprat de Figueiredo - FUNDACENTRO; do II Workshop Internacional "Policiamento em campo e testes para avaliação de motoristas sob efeito de álcool e drogas" do Departamento de Medicina Legal, Ética Médica e Medicina Social e do Trabalho da Faculdade de Medicina da Universidade de São Paulo; Simpósio de Aplicação Multiprofissional: "Transtornos Mentais no Trabalho" - Instituto de Psicologia Aplicada e Formação (IPAF).

Todos os médicos residentes freqüentam as aulas do Curso de Especialização em MT, que desde 2004 é realizado no período noturno as segundas, terças e quartas feiras entre as $19 \mathrm{~h}$ e $23 \mathrm{~h}$ durante um ano letivo.

A Tabela 6 relaciona os módulos com as respectivas disciplinas e cargas horárias do curso de especialização para o primeiro ano, que totaliza uma carga horária de 584 horas. 
Tabela 6. Disciplinas e respectiva carga horária do primeiro ano do curso de especialização em MT da FMUSP, 2004-2010

\begin{tabular}{|c|c|c|}
\hline Módulo & Disciplina & Horas \\
\hline \multirow{7}{*}{$\mathbf{I}$} & DA MEDICINA DO TRABALHO À SAÚDE DO TRABALHADOR & \\
\hline & Introdução à saúde do trabalhador & $16 \mathrm{~h}$ \\
\hline & Ética em saúde do trabalhador & $12 \mathrm{~h}$ \\
\hline & Sociologia do trabalho & $20 \mathrm{~h}$ \\
\hline & Metodologia de pesquisa científica & $20 \mathrm{~h}$ \\
\hline & Bioestatística & $24 \mathrm{~h}$ \\
\hline & Epidemiologia & $28 \mathrm{~h}$ \\
\hline \multirow{5}{*}{ II } & BASES PARA ESTUDO DA SAÚDE DO TRABALHADOR & \\
\hline & Legislação aplicada á saúde dos trabalhadores & $20 \mathrm{~h}$ \\
\hline & Segurança do trabalho & $24 \mathrm{~h}$ \\
\hline & Ergonomia & $40 \mathrm{~h}$ \\
\hline & Organização do trabalho e saúde & $12 \mathrm{~h}$ \\
\hline \multirow{5}{*}{ III } & AGENTES FÍSICOS E QUÍMICOS E PROTEÇÃO A SAÚDE & \\
\hline & Higiene do trabalho & $28 \mathrm{~h}$ \\
\hline & Patologia: os agentes físicos & $32 \mathrm{~h}$ \\
\hline & Toxicologia & $28 \mathrm{~h}$ \\
\hline & Patologia: os agentes químicos & $36 \mathrm{~h}$ \\
\hline \multirow{9}{*}{ IV } & SERVIÇOS DE SAÚDE DOS TRABALHADORES & \\
\hline & Outras patologias & $24 \mathrm{~h}$ \\
\hline & Saúde mental e trabalho & $24 \mathrm{~h}$ \\
\hline & Saúde ambiental & $16 \mathrm{~h}$ \\
\hline & Organização de serviços de saúde do trabalhador & $24 \mathrm{~h}$ \\
\hline & Administração e planejamento & $16 \mathrm{~h}$ \\
\hline & ATIVIDADES PRÁTICAS & $60 \mathrm{~h}$ \\
\hline & Visitas a empresas & \\
\hline & MONOGRAFIA/TRABALHO DE CONCLUSÃO DE CURSO & $80 h$ \\
\hline TOTAL & & $584 h$ \\
\hline
\end{tabular}

Fonte: Arquivos do Departamento de Medicina Legal, Ética Médica, Medicina Social e do trabalho da FMUSP, 2004-2010.

\section{DISCUSSÃO}

A Residência Médica em Medicina do Trabalho na FMUSP é recente, tendo formado somente seis médicos do trabalho no período analisado neste estudo, ocorrendo diversas mudanças no programa ao longo dos anos, como forma de adaptação e melhoria. De forma geral, o programa além de preencher e superar quantitativa e qualitativamente os requisitos mínimos da CNRM, privilegiando os estágios nas áreas clínicas, tendo como ponto positivo a estrutura e a diversidade de especialidades do Hospital das Clínicas da FMUSP.

Os estágios em empresas públicas e privadas, sindicatos de trabalhadores e em instituições periciais foram variados, conforme disponibilidade dos seus profissionais responsáveis e por vínculos pessoais do supervisor da residência médica, sem um critério 
claro para inclusão e exclusão desses estágios.

Com relação aos estágios clínicos, todos os residentes passaram pelos ambulatórios de especialidades e serviços de atendimento de urgência requeridos no programa. Os estágios de neurologia e oftalmologia, por sua vez, foram introduzidos a partir de 2007 e após avaliação dos residentes, foram incorporados para melhorar o programa.

Mudanças na grade curricular também são feitas em outros programas de Residência em Medicina do Trabalho ao longo dos anos, para melhor adequar a formação do residente à especialidade. Na Santa Casa de São Paulo, por exemplo, um dos dois meses do estágio em emergências clínicas foi substituído por um mês no pronto-socorro de cirurgia em 2005, e em 2007 houve mudança do estágio de enfermaria da clínica médica para o ambulatório do mesmo serviço ${ }^{10}$.

Os estágios em serviços de saúde de empresas e sindicatos de trabalhadores tiveram como característica a diversidade e a busca de diferentes ramos de atividade econômica. Cada residente teve a oportunidade de conhecer pelo menos quatro diferentes ramos de atividade, porém em cada ano, as empresas não foram necessariamente as mesmas. Não existem convênios com as empresas para os estágios, sendo que a seleção destas é feita de maneira informal.

Nos últimos três anos, houve uma tendência de se transferir os estágios para empresas prestadoras de serviço, em detrimento do setor de metalurgia, devido principalmente ao crescimento do setor terciário e a incorporação de antigos residentes e ex-alunos do Curso de Especialização de MT da FMUSP por essas empresas, o que tem facilitado a sua inclusão nas últimas grades de estágios do período em análise.

No setor público, todos os residentes passaram em órgãos do MTE e Serviços de Saúde do Trabalhador do MS. Com relação aos órgãos relacionados à perícia médica (INSS e IMESC), somente alguns residentes realizaram estágio nesses órgãos. Outras Residências de Medicina do Trabalho relatam a mesma dificuldade de estágio nessas instituições, que não autorizam oficialmente programas para médicos residentes de Medicina do Trabalho ${ }^{9,10}$. Estes estágios são feitos mediante contatos com os responsáveis desses órgãos públicos, baseados no conhecimento individual dos supervisores dos programas.

Nesse estudo verificamos que a formação dos Médicos do Trabalho não pode ser completa sem a inclusão de áreas práticas em empresas e órgãos públicos que, após sua formação, irão absorver esses profissionais. É extremamente importante a oficialização dos estágios junto a esses órgãos em todo o Brasil. A solução seria a conjunção de esforços entre os diversos programas de residência de MT em todo o país, para que os órgãos públicos incluam estes estágios nos seus programas de atividades de forma oficial.

Durante os seis anos de existência do Programa de Residência de Medicina do Trabalho da FMUSP, foi notória a falta de estágios em instituições que dêem ao médico residente uma visão global sobre administração e gestão de serviços. No âmbito das suas competências, espera-se que o médico do trabalho assuma responsabilidades quer como médico coordenador de programas de controle médico de saúde ocupacional quer em programas de qualidade de vida e/ou de gestão de serviços especializados de saúde e segurança no trabalho em vários setores do mercado de trabalho.

O programa de atividades teórico-práticas da FMUSP está de acordo com o programa mínimo da CNRM, no que diz respeito às disciplinas teóricas e carga horária ministradas no Curso de Especialização de Medicina do Trabalho. O Curso de Especialização inclusive conta com aulas ministradas por profissionais do Serviço de Saúde Ocupacional (SSO) do Hospital das Clínicas (HC) da FMUSP, que está vinculado à Disciplina de Medicina Social e do Trabalho, contribuindo para uma formação mais voltada para a prática, devido à sólida experiência desses profissionais na área.

Essas atividades teóricas ao longo dos anos vêm sendo complementadas com discussões em reuniões semanais, onde são trazidos casos clínicos, artigos científicos e relatos da prática da Medicina do Trabalho por convidados como forma de consolidar conhecimentos e ter um olhar crítico sobre as normas técnicas, protocolos de procedimentos e padronização de rotinas sobre questões de saúde relacionadas ao trabalho ${ }^{7}$.

Ao longo das últimas décadas ocorreram mudanças no padrão de adoecimentos dos trabalhadores, decorrentes de vários fatores. Dias et al. ${ }^{6}$ enumeram alguns destes fatores: transformações nos processos de produção; introdução de novas tecnologias no mercado de trabalho; novas modalidades de contratos e relações de trabalho; novas modalidades de organização e gestão do trabalho e maior preocupação com a saúde dos trabalhadores, consubstanciada na melhoria do arcabouço jurídico-institucional da área de saúde e segurança; aumento das fiscalizações dos locais 
de trabalho; melhores diagnósticos resultantes do maior conhecimento das patologias relacionadas ao trabalho; aumento dos movimentos sociais organizados de trabalhadores, reivindicando por melhores condições de trabalho, qualidade de vida e exercícios dos direitos humanos básicos; maior e melhor capacidade de negociação e formulação de consensos entre empregados e empregadores; surgimentos de programas de qualidade e certificação e de gestão integrada de saúde, segurança e meio ambiente.

Como exemplo, na década de 80, prevaleciam os agravos relativos ao ruído, com as perdas auditivas induzidas pelo ruído (PAIR) e na década de 90, as lesões por esforços repetitivos (LER) assumiram a liderança das patologias relacionadas ao trabalho.

No século XXI, fruto de maiores exigências individuais e demandas multiprofissionais relativas a metas de produção, disputas de mercado, formas de organização do trabalho, insegurança no emprego, enxugamento no quadro de funcionários das empresas, incorporação tecnológica, falta de suporte social entre outros, assinala-se um aumento cada vez maior de patologias mentais relacionadas ao trabalho.

Todas estas mudanças justificam adaptações, melhorias e mudanças para a otimização dos programas de formação do profissional Médico do Trabalho, quer em forma de cursos de especialização, como na modalidade de residência médica ${ }^{6}$.

O intercâmbio entre os diversos programas espalhados pelo país, a identificação de semelhanças, a partilha de sucessos e fracassos e a busca de soluções para os problemas comuns, poderia ser uma forma de aperfeiçoamento contínuo e uniforme dos programas de residência médica de medicina do trabalho no Brasil.

O acompanhamento e supervisão dos estágios dos residentes de Medicina do Trabalho da FMUSP poderiam ser melhorados com a definição de critérios mais claros de avaliação, sobretudo nos estágios em empresas e órgãos públicos. O modelo de avaliação usado para as práticas nas áreas clínicas pareceu-nos inadequado para avaliação dos estágios em empresas e órgãos públicos, uma vez que as atividades nestes estágios referem-se a outras competências para o médico do trabalho, distintas daquelas relacionadas às áreas clínicas.

A preceptoria do departamento é feita de forma alternada entre a medicina do trabalho e a medicina legal. Para uma melhor organização, elaboração das grades anuais de estágios, programação das atividades teóricas e práticas, supervisão nos locais de estágio, contato regular com os responsáveis pelos estágios nas empresas dentre outras, tornase necessária uma discussão e melhor articulação entre as duas residências, para que a supervisão dos preceptores seja uniforme para os residentes da Medicina do Trabalho e da Medicina Legal.

Durante a realização deste estudo foram notórios alguns problemas relacionados à estrutura administrativa da residência. A falta de uma secretária específica da residência de Medicina do Trabalho, à semelhança do que acontece com os outros cursos que funcionam no Departamento de Medicina Legal, Ética Médica e Medicina Social e do Trabalho, é uma dificuldade que, sanada, resultaria em melhor organização administrativa, principalmente quanto ao registro de dados e informações das atividades dos docentes, preceptores e residentes e o arquivo adequado da documentação.

No decorrer do estudo sentimos igualmente a falta de uma análise periódica da evolução cronológica do Programa de Residência Médica de Medicina do Trabalho da FMUSP, com o registro de informações relacionadas às diferentes mudanças nas grades e locais de estágios, dificuldades, progressos e retrocessos no programa, como forma de criar um histórico desta evolução e servir de fonte de informação para trabalhos como este. A iniciativa da realização desta análise pode servir de incentivo para trabalhos semelhantes futuros.

Este estudo foi feito com objetivo acadêmico e constituiu um momento importante para análise dos avanços e retrocessos do Programa de Residência de Medicina do Trabalho da FMUSP, desde a sua institucionalização em 2004 até 2010. Neste período, importantes transformações ocorreram nos seus programas e grades curriculares anuais, visando a formação de profissionais com competências necessárias para atuar na área de saúde e segurança dos trabalhadores em todo o país.

Apesar dos grandes avanços registrados ao longo do período de estruturação de um programa que faça jus às necessidades do mercado de trabalho cada vez mais exigentes pelas constantes mudanças no perfil de organização e adoecimento dos trabalhadores, existem ainda várias oportunidades de melhoria.

\section{CONCLUSÕES}

Após análise sobre o Programa de Residência Médica em Medicina do Trabalho da FMUSP relativamente ao processo que culminou com o seu credenciamento e a evolução das grades curriculares 
modificadas ao longo do período de 2004 à 2010, podemos concluir que:

$>$ De uma forma geral, o Programa de Residência de Medicina do Trabalho da FMUSP preenche os requisitos mínimos da CNRM para a formação de médicos do trabalho competentes para atuar na área de saúde e segurança dos trabalhadores no Brasil e ao longo do período em análise sofreu várias alterações na sua estrutura curricular visando melhorias e adaptações á realidade da problemática da saúde e segurança do trabalhador;

$>$ Os estágios clínicos dos médicos residentes concentram-se nos diferentes ambulatórios e serviços de urgência do Hospital das Clínicas da FMUSP. Outros locais de estágio foram empresas de diferentes ramos de atividade (hospitalar, metalúrgico, petroquímico, automobilístico, serviços de saúde de sindicatos de trabalhadores), bem como em serviços públicos como a Superintendência Regional do Trabalho e Emprego (SRT), a Fundação Jorge Duprat de Figueiredo (FUNDACENTRO), a Secretaria de Estado da Saúde (SES), os Centros de Referência em Saúde do Trabalhador (CEREST e CRST) e o Centro de Saúde Escola (CSE).

$>$ As aulas teóricas no curso de especialização de MT da FMUSP, as discussões de casos clínicos e artigos científicos sobre a saúde dos trabalhadores, estágios optativos, seminários, congressos e simpósios são as atividades teórico-práticas do programa de residência médica de MT da FMUSP.

Agradecimentos: à Escola de Educação Permanente do Hospital das Clínicas da Faculdade de Medicina da Universidade de São Paulo, gerenciadora do "Programa Angola - Clínica Multiperfil" que realiza a capacitação de médicos angolanos no Brasil; a Profa. Maria Lúcia Bueno Garcia da Clínica Médica do HCFMUSP pelo incentivo e ao Dr. Cristian Kotinda Junior, aos professores, assistentes, colegas e funcionários do Departamento de Medicina Legal, Ética Médica, Medicina Social e do Trabalho da FMUSP pela colaboração durante a coleta de dados e redação da monografia de conclusão da Residência Médica de Medicina do Trabalho que serviu de precursor para a elaboração deste artigo.

\section{REFERÊNCIAS}

1. Brasil. Lei $n^{\circ} 6.932$, de 7 de julho de 1981. Dispõe sobre as atividades do médico residente e dá outras providências Brasília; 1981 [citado 06 jul. 2010]. Disponível em: http://www.planalto.gov.br/ccivil_03/ leis/L6932.htm.

2. Sousa EG. A residência médica em pneumologia no Brasil. J Bras Pneumol. 2004;30(2):253-9.

3. Organización Panamericana de La Salud (OPS). Residencias médicas en América Latina. Washington: OPS; 2011. (Serie: La Renovación de La Atención Primaria de Salud en la América $n^{\circ} 5$ ).

4. Mendes R, Dias EC. Da medicina do trabalho à saúde do trabalhador. Rev Saúde Pub (S. Paulo). 1991;25(5):341-9.

5. Dias EC, Mendes R. A medicina do trabalho: suas origens e campo de atuação. In: D’Assumpção EA. Livro das especialidades médicas. Belo Horizonte: Coopmed; 2000. p.109-15.

6. Dias EC, Gontijo ED, Oliveira RB. Formação, educação continuada e certificação em medicina do trabalho: uma proposta orientada pelas competências requeridas para o exercício profissional. Rev Bras Med Trab. 2003;1(1):6-21.

7. Silveira AM, Dias EC. A formação do médico do trabalho: residência médica em foco. Rev Bras Med Trab. 2004;2(1):4-10.

8. Associação Nacional de Medicina do Trabalho (ANAMT). Conhecimento - Medicina do trabalho reconhecimento da especialidade - obtenção do título de especialista em MT [citado 11 jul. 2011]. Disponível em: http://www.anamt.org.br/index.php?id_materia=24

9. Dutra GSP, De Pádua CHS, Bim CM, Navarro EMRL, Moreira AAG, De Almeida FSS, De Freitas JBP, Morrone LC. A residência em medicina do trabalho na Santa Casa de São Paulo - 2007. In: XXII Jornada Paranaense de Saúde Ocupacional; out. 4-6; Curitiba, PR, 2007.

10. Hayashide JM, De Oliveira OAC, Coelho GM, De Arruda LM, Borguetti FC, Morrone LC. Experiência dos Programas de Residência em Medicina do Trabalho em dois hospitais de ensino de São Paulo. Rev Bras Med Trab. 2010;8(1):23-32.

11. Brasil. Ministério da Saúde. Saúde do trabalhador. Cadernos de atenção básica $n^{\circ} 5$. Brasília, DF: Secretaria de Políticas de Saúde; 2002.

Artigo recebido em: 04/05/2011

Artigo aceito em: 20/07/2011 